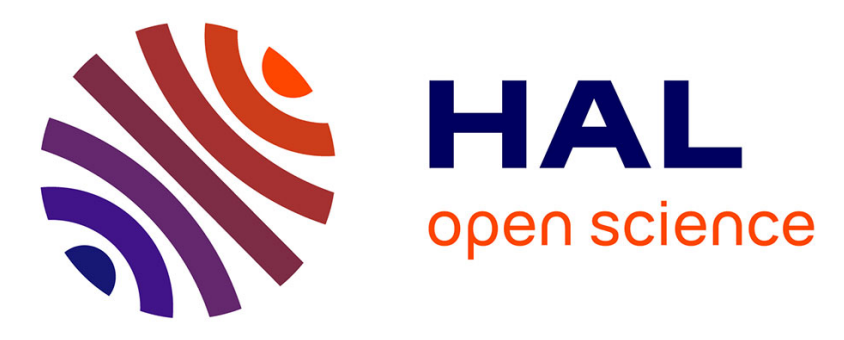

\title{
Time resolved terahertz spectroscopy of low frequency electronic resonances, and optical pump-induced terahertz photoconductivity in reduced graphene oxide membrane
}

\author{
Srabani Kar, Swetha Jayanthi, Eric Freysz, Ajay Kumar Sood
}

\section{To cite this version:}

Srabani Kar, Swetha Jayanthi, Eric Freysz, Ajay Kumar Sood. Time resolved terahertz spectroscopy of low frequency electronic resonances, and optical pump-induced terahertz photoconductivity in reduced graphene oxide membrane. Carbon, 2014, 80, pp.762-770. 10.1016/j.carbon.2014.09.030 . hal-01094641

\section{HAL Id: hal-01094641 \\ https://hal.science/hal-01094641}

Submitted on 19 Dec 2014

HAL is a multi-disciplinary open access archive for the deposit and dissemination of scientific research documents, whether they are published or not. The documents may come from teaching and research institutions in France or abroad, or from public or private research centers.
L'archive ouverte pluridisciplinaire HAL, est destinée au dépôt et à la diffusion de documents scientifiques de niveau recherche, publiés ou non, émanant des établissements d'enseignement et de recherche français ou étrangers, des laboratoires publics ou privés.

\section{(ㄷ)(1) $\$$}

Distributed under a Creative Commons Attribution - NonCommerciall 4.0 International 


\title{
Time resolved terahertz spectroscopy of low frequency electronic resonances and optical pump-induced terahertz photoconductivity in reduced graphene oxide membrane
}

\author{
Srabani Kar ${ }^{a, b}$, Swetha Jayanthi ${ }^{c}$, Eric Freysz ${ }^{d}$, A.K. Sood ${ }^{a, b, *}$ \\ a Department of Physics, Indian Institution of Science, Bangalore 560 012, India \\ b Center for Ultrafast Laser Applications, Indian Institution of Science, Bangalore 560 012, India \\ c Centre for Nano Science and Engineering, Indian Institution of Science, Bangalore 560 012, India \\ 'd University of Bordeaux, LOMA, UMR CNRS 5798, 351, Cours de la liberation, 33405 Talence cedex, France
}

\begin{abstract}
A B S T R A C T
Towards ultrafast optoelectronic applications of single and a few layer reduced graphene oxide (RGO), we study time domain terahertz spectroscopy and optical pump induced changes in terahertz conductivity of self supported RGO membrane in the spectral window of $0.53 .5 \mathrm{THz}$. The real and imaginary parts of conductivity spectra clearly reveal low frequency resonances, attributed to the energy gaps due to the van Hove singularities in the density of states flanking the Dirac points arising due to the relative rotation of the graphene layers. Further, optical pump induced terahertz conductivity is positive, pointing to the dominance of intraband scattering processes. The relaxation dynamics of the photo excited carriers consists of three cooling pathways: the faster ( $450 \mathrm{fs})$ one due to optical phonon emission followed by disorder mediated large momentum and large energy acous tic phonon emission with a time constant of a few ps (called the super collision mecha nism) and a very large time ( $100 \mathrm{ps})$ arising from the deep trap states. The frequency dependence of the dynamic conductivity at different delay times is analyzed in term of Drude Smith model.
\end{abstract}

\section{Introduction}

Graphene is a $s p^{2}$ hybridized 2D honeycomb lattice with elec tronic linear band dispersion having zero band gap at $\mathrm{K}$ and $\mathrm{K}^{\prime}$ points of the Brillouin Zone. The carriers act like massless Dirac Fermions characterized by Fermi velocity $v_{\mathrm{F}} \approx 1.1 \times 10^{6} \mathrm{~m} / \mathrm{s}$. These special attributes give rise to unusual electronic properties such as high carrier mobility,
Klein paradox and anomalous integer quantum Hall effect at room temperature [1 7]. It has been recognized that graph ene is a very attractive platform for potential applications in electronics and optoelectronics devices such as terahertz modulators [8], solar photovoltaic cells [9,10], mode locked lasers [11], ultrafast photodetectors [12] etc. Therefore the production of low cost large amount of graphene becomes an important issue. The highest quality graphene devices

\footnotetext{
* Corresponding author at: Department of Physics, Indian Institution of Science, Bangalore 560012 , India.

E mail address: asood@physics.iisc.ernet.in (A.K. Sood).
} 
are still being made using mechanical exfoliation technique. The quality of graphene synthesized using chemical vapor deposition and epitaxially grown graphene on $\mathrm{SiC}$ is getting better with time. In recent years the ultrasonic assisted exfolia tion of graphite to get a few layer graphene [13] and chemically produced reduced graphene oxide (RGO) by reduction of exfoli ated graphene oxide (GO) $[14,15]$ have been shown to be very promising routes for low cost large scale production. To realize RGO based high performance optoelectronic devices, discerning the effects of disorder and layer misorientation on the conduc tivity of RGO is an important issue. Terahertz (THz) spectral regime is of particular interest in case of graphene since it directly probes the carrier dynamics close to the Dirac point.

$\mathrm{THz}$ conductivity using time domain spectroscopy of chemical vapor deposited and epitaxial grown monolayer graphene has been previously reported ${ }^{1}$ [16 19]. Similar mea surements on reduced graphene oxide network films depos ited on quartz substrates have also been carried out, which showed an increase of $\mathrm{THz}$ conductivity by reducing graphene oxide at higher temperatures [20]. Broadband optical absorp tion measurements from terahertz to visible frequency range have been used to get information about the number of layers and carrier density [21 24]. The optical response of back gated graphene has also been studied from infra red to $\mathrm{THz}$ region $[25,26]$. All these experimental measurements have shown Drude like frequency dependence of the conductivity from $\mathrm{THz}$ to mid infrared range. In comparison to Drude conductiv ity, plasmon related resonant features have been recently reported in CVD grown monolayer graphene [27]. Zou et al. [28] have shown a band at $\sim 2.7 \mathrm{THz}$ in the conductivity spectra of twisted bilayer graphene, attributed to the interband transi tion across the energy gap arising from relative layer rotation induced van Hove singularity in the density of states. The tun neling differential conductance measurements do show the presence of rotation induced van Hove singularities flanking at different energies, depending on the angle of rotation in twisted graphene layers [29]. RGO is an interesting system with graphene like electronic band structure affected by the presence of large disorder and misorientation in layer stack ing. Thus the conductivity and carrier relaxation in RGO are expected to be strongly influenced by the defects and the mod ified electronic structure. Very recently, the effect of trap den sity on carrier relaxation pathways in graphene oxide have been studied in the $\mathrm{THz}$ regime showing that the Auger recombination and trap assisted dynamics play a crucial role in cooling of the photoexcited carriers [30]. In contrast, tran sient absorption experiments [31] have shown that optical phonon emission and trap assisted cooling in RGO may dom inate. Thus, the issue of non equilibrium carrier dynamics is still not understood well in RGO.

In this work, we report time resolved terahertz spectros copy of self supported RGO membrane in the spectral range of $0.53 .5 \mathrm{THz}$, clearly revealing three resonances in the real and imaginary parts of terahertz conductivity. These have been assigned to the enhanced density of states due to the layer rotation induced van Hove singularities at the saddle points in electronic dispersion of twisted two graphene layers in RGO [28]. The electronic part of the complex conductivity spectra is explained in terms of Drude Smith model incorpo rating the localization of carriers due to defects. Optical pump terahertz probe (OPTP) experiments show positive dynamic conductivity due to the dominance of intraband scattering processes. After pumping, the hot carriers ther malize generally within a few tenths of femtoseconds to a quasi Fermi Dirac distribution with electron temperature $T_{e}$. Afterwards, the hot carriers cool down via different scattering mechanisms like Auger scattering, plasmon emission, optical phonon emission, acoustic phonon emission etc. We observe three relaxation times: fast component with time constant of $\sim 450 \mathrm{fs}$ due to emission of optical phonons, a slower component ( $\sim$ a few ps decay time) due to disorder mediated carrier acoustic phonon scattering and a very weak and slow component ( 100 ps). The frequency dependence of the dynamic conductivity is also measured and explained in terms of Drude Smith model. The fitted parameters lead to the conclusion that after pump excitation the carriers gain enough energy to cross the nearby barriers resulting in higher average momentum relaxation time and provide an important view of the cooling dynamics of photoexcited carriers in RGO.

\section{Experimental details}

\subsection{Sample preparation and characterization}

Graphene oxide was prepared using simplified Hummer's method [15]. Briefly, graphite flakes (2 g) were added to a mix ture of $\mathrm{H}_{2} \mathrm{SO}_{4}(110 \mathrm{ml})$ and $\mathrm{H}_{3} \mathrm{PO}_{4}(12 \mathrm{ml})$. After stirring the mixture for some time $\mathrm{KMnO}_{4}(11.1 \mathrm{~g})$ was added slowly, fol lowed by stirring at room temperature for 3 days and then adding $\mathrm{H}_{2} \mathrm{O}_{2}(20 \mathrm{ml})$. Graphite oxide formed was washed repeatedly with $1 \mathrm{M} \mathrm{HCl}$ and distilled water, until the $\mathrm{pH}$ of the solution was 34 . The resulting solution was centrifuged at $10,000 \mathrm{rpm}$ to separate graphene oxide (GO) which was reduced chemically by two methods to get reduced graphene oxide: one using hydrazine hydrate (denoted by $\mathrm{RGO}_{\mathrm{Hz}}$ ) and the other using hydroiodic (HI) acid (denoted by $\mathrm{RGO}_{\mathrm{HI}}$ ). For hydrazine method [14], GO (50 mg) was dispersed in water $(100 \mathrm{ml})$ and centrifuged at $3000 \mathrm{rpm}$ for $30 \mathrm{~min}$ to remove any un exfoliated GO. Hydrazine hydrate $(30 \mu \mathrm{L}, 64 \mathrm{wt} \%$ in water) and ammonia solution $(0.8 \mathrm{ml}, 25 \%)$ were added to the above dispersion. The resulting mixture was stirred at $100{ }^{\circ} \mathrm{C}$ for $5 \mathrm{~h}$. The solution was centrifuged at 10,000 rpm to obtain $\mathrm{RGO}_{\mathrm{Hz}}$, following by filtering the dispersion of $\mathrm{RGO}_{\mathrm{HZ}}$ in DMF (Dimethylformamide) through filter paper assisted by a vacuum pump. For the HI reduction, the procedure is as follows [32]: GO (100 mg) was dispersed in acetic acid $(38 \mathrm{ml})$ followed by addition of the $\mathrm{HI}(2 \mathrm{ml})$ and stirring for $40 \mathrm{~h}$ at $40^{\circ} \mathrm{C}$. This was followed by centrifuging and washing the product with sodium bi carbonate, de ionized water and acetone. The average thickness of $\mathrm{RGO}_{\mathrm{HZ}}$ is $\sim 370 \mathrm{~nm}$ and $\mathrm{RGO}_{\mathrm{HI}}$ is $\sim 15 \mu \mathrm{m}$.

\footnotetext{
${ }^{1}$ Kar S., Mohapatra D. R., Freysz E., Sood A. K. Tuning photoinduced terahertz conductivity in monolayer graphene: Optical pump terahertz probe spectroscopy 2014;arXiv:1407.2752
} 


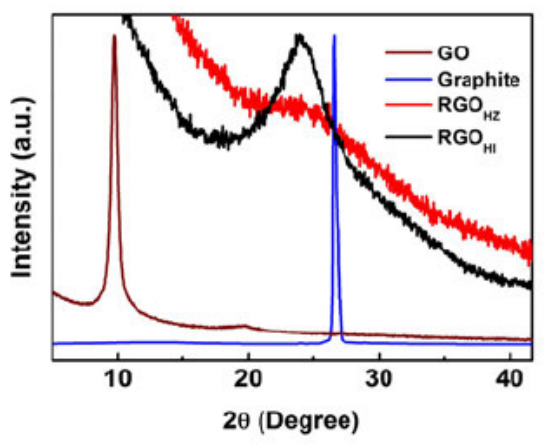

(a)

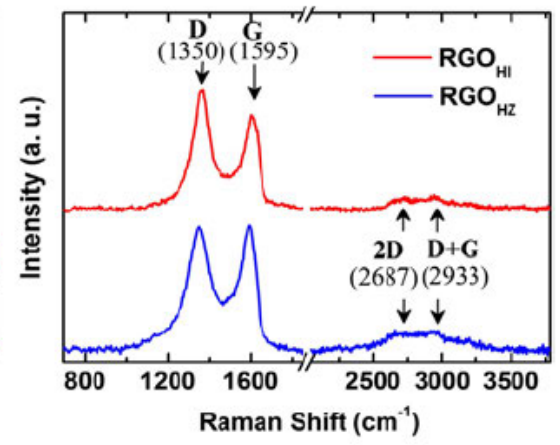

(b)

Fig. 1 - (a)X-ray diffraction patterns of Graphite (blue solid line), graphene oxide (GO) (wine solid line), $\mathrm{RGO}_{\mathrm{Hz}}(\mathrm{red}$ solid line) and $\mathrm{RGO}_{\mathrm{HI}}$ (black solid line). (b) Raman spectra of $\mathrm{RGO}_{\mathrm{Hz}}$ (blue solid line) and $\mathrm{RGO}_{\mathrm{HI}}$ (red solid line). (A color version of this figure can be viewed online.)

The samples were characterized by UV visible spectros copy, Raman spectroscopy, atomic force microscopy and $\mathrm{X}$ ray diffraction. The suspension has mostly single layer graph ene with average lateral size of $2 \mu \mathrm{m}$. Fig. 1a shows $\mathrm{X}$ ray dif fraction pattern revealing that the $\mathrm{RGO}_{\mathrm{Hz}}$ has a broad peak at $2 \theta \sim 25^{\circ}$ (d spacing $\sim 3.56 \AA$ ) which correspond to the partial restacking of exfoliated graphene layers with two dimen sional sheets along [002] direction. A more prominent peak at $2 \theta \sim 25^{\circ}$ is observed for $\mathrm{RGO}_{\mathrm{HI}}$ (Fig. 1a) indicating better quality graphene. In comparison, graphite exhibits a sharp peak at $2 \theta 26.5^{\circ}$, corresponding to the interlayer distance $\sim 3.34 \AA$ and GO shows a peak at $2 \theta 9.7^{\circ}$ (d spacing $\sim 9.11 \AA$ ) due to oxygen containing functional group and intercalated water molecules. Fig. $1 \mathrm{~b}$ shows the Raman spec tra of the $\mathrm{RGO}_{\mathrm{Hz}}$ and $\mathrm{RGO}_{\mathrm{HI}}$ membranes recorded at room temperature in backscattering geometry using $\lambda 514 \mathrm{~nm}$ laser light and WItec Raman spectrometer. Raman bands are observed at $1595 \mathrm{~cm}^{1}$ ( $\mathrm{G}$ band), $1350 \mathrm{~cm}^{1}$ (D band) and $2687 \mathrm{~cm}^{1}$ (2D band). The size of defect free domain, $L_{D}$, can be estimated by using the intensity ratios of the $D$ and $G$ bands [33]: $\mathrm{L}_{\mathrm{D}}^{2}\left(\mathrm{~nm}^{2}\right) \quad(1.8 \pm 0.5) \times 10^{9} \lambda^{4}\left(\mathrm{I}_{\mathrm{D}} / \mathrm{I}_{G}\right)^{1}$ giving $L_{D} \sim 11 \pm 2 \mathrm{~nm}$ for $\mathrm{RGO}_{\mathrm{HZ}}$ and $\sim 8 \pm 2 \mathrm{~nm}$ for $\mathrm{RGO}_{\mathrm{Hl}}$.

\subsection{THz experimental set up}

$\mathrm{THz}$ radiation is generated via two color laser filament using Ti: Sapphire laser delivering 70 fs pulse of maximum energy $\sim 1.6 \mathrm{~mJ}$ per pulse at a central wavelength of $785 \mathrm{~nm}$ with rep etition rate $1 \mathrm{kHz}$. The beam is divided into three parts: (1) to generate terahertz, (2) to probe terahertz and (3) for pumping the sample. The pump excitation energy is varied by using neutral density filters to give a fluence varying from 3.4 to $340 \mu \mathrm{J} / \mathrm{cm}^{2}$. The fundamental laser beam and its second har monic laser beams are focused in air to produce plasma. Ter ahertz radiation generated from the plasma [34] is focused at the sample position by using a pair of off axis parabolic mirrors of $10 \mathrm{~cm}$ focal length. The unwanted light generated from the plasma is blocked by $1 \mathrm{~mm}$ thick high resistive silicon wafer. After propagating through the sample the tera hertz beam is collected and focused at $1 \mathrm{~mm}$ thick ZnTe detector by a pair of parabolic mirrors. The whole terahertz beam path was purged with dry nitrogen to avoid unwanted absorptions by water vapors. Terahertz time domain mea surements were performed in transmission geometry. Photo induced changes in terahertz transmission due to optical pump at $785 \mathrm{~nm}$ was measured at the peak position of the ter ahertz field. Fig. 2 shows a schematic of the experimental set up to perform optical pump terahertz probe transmission measurements. While measuring the terahertz field passing through unexcited sample, the chopper was placed in the ter ahertz generation path and the gate delay line $t_{d}$ was used. In case of the photoinduced transmission measurements, the chopper was placed in the path of the pump beam exciting the RGO membrane and the pump delay line $t_{p}$ was used.

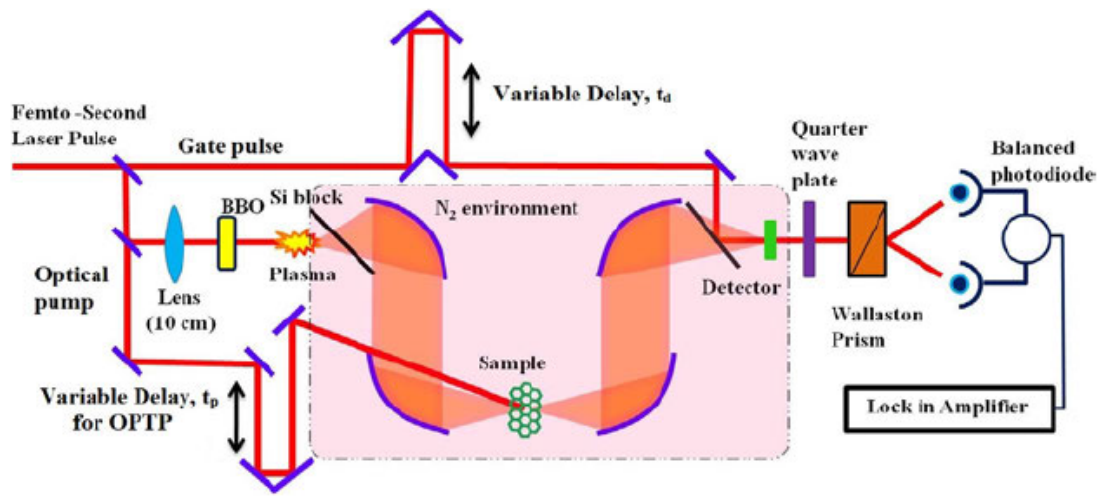

Fig. 2 - Schematic of the THz experimental set up for time resolved terahertz spectroscopy and optical pump-terahertz probe spectroscopy. (A color version of this figure can be viewed online.) 
The spot size of the terahertz beam on the sample position was $2 \mathrm{~mm}$ while the size of pump beam $(785 \mathrm{~nm})$ to excite the sample was $5 \mathrm{~mm}$. The estimated terahertz peak field was $\sim 26 \mathrm{kV} / \mathrm{cm}$.

\section{Results and discussion}

\subsection{THz conductivity measurements}

Fig. 3a shows the terahertz electric field through air $E_{\text {ref }}(t)$ and through the RGO membrane $E_{\text {sam }}(t)$. After taking the Fourier Transform, the spectral function is evaluated as $T(\omega)$ $\frac{E_{\text {sam }}(\omega)}{E_{\text {rff }}(\omega)} \quad \mathrm{A}(\omega) \exp (i \theta(\omega))$, where $\mathrm{A}(\omega)$ is the amplitude and $\theta(\omega)$ is the phase of the transmission function given by $[35,36]$
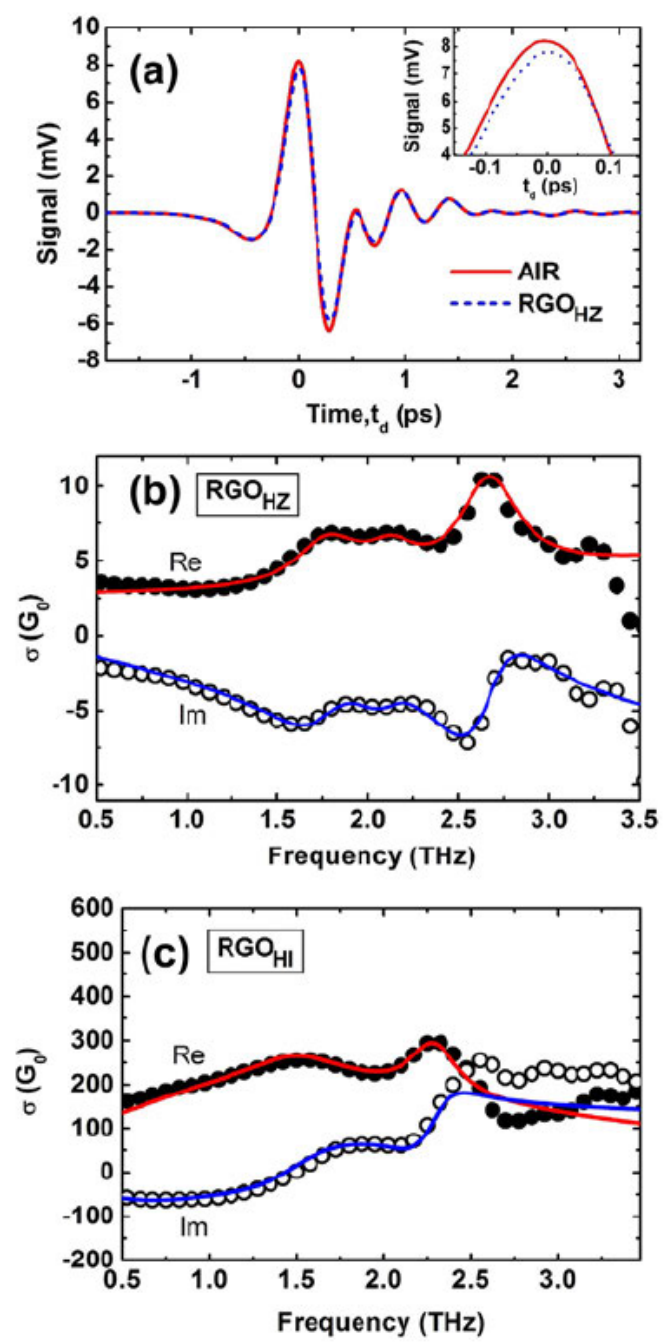

Fig. 3 - (a) Terahertz time domain signal through the $\mathbf{R G O}_{\mathrm{Hz}}$ membrane (blue dotted line) and air (continuous line). Inset: enlarged part of the THz pulse near the maximum. (b,c) Real and imaginary part of the conductivity spectra. Filled circles represent real part of conductivity and open circles represent imaginary part. The solid lines represent the fitted curves of the Drude-Smith model along with the Lorentz model. (A color version of this figure can be viewed online.)

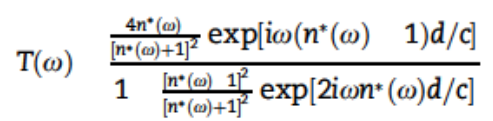

where $n^{*}(\omega)$ is the complex refractive index of the membrane, $\mathrm{d}$ is the thickness of the membrane and $\mathrm{c}$ is velocity of light. In the limit $n^{*} \omega d / c \ll 1$, one can approximate Eq. (1) for the membrane as $T(\omega) \quad \frac{2}{2+Z_{0 \sigma(\omega)}}$, where $Z_{0} \quad 377 \Omega$ is impedance of free space and $\sigma(\omega)$ is complex conductivity of the membrane. Now we will present the results of $\mathrm{RGO}_{\mathrm{HZ}}$. Fig. $3 \mathrm{~b}$ shows the real (filled circles) and imaginary part (open circles) of the complex conductivity of the $\mathrm{RGO}_{\mathrm{Hz}}$ membrane in the spectral range of 0.5 to $3.5 \mathrm{THz}$ in units of $\mathrm{G}_{0}\left(2 \mathrm{e}^{2} / \mathrm{h} \quad 77.3 \mu \mathrm{S}\right)$. It can be noted that the imaginary part of conductivity is negative throughout the whole spectral range and three resonances are clearly discernible at 1.8, 2.2 and $2.7 \mathrm{THz}$ in the real and imaginary parts of the terahertz conductivity. We find that Drude model used for single layer graphene $[16,17,20]$ cannot account for the contribution of the carriers to $\sigma(\omega)$. One should note that the graphene basal planes of GO are decorated non uniformly by oxygen contain ing functional groups (mainly epoxy and hydroxyl). The pres ence of such functional groups results in small ordered $s p^{2}$ clusters isolated within $s p^{3}$ C O matrix [37]. The thermal reduction of GO creates new $s p^{2}$ domains, which provide per colation pathways between $s p^{2}$ clusters [37]. These new $s p^{2}$ clusters can provide hopping pathways for the carrier trans port. Such localized finite size molecular $s p^{2}$ clusters embed ded within $s p^{3}$ matrix lead to confinement of $\pi$ electrons in RGO. Drude Smith model takes into account such carrier localization [38]. Accordingly, the complex conductivity spectra shown in Fig. $3 \mathrm{~b}$ has been fitted using

$\sigma \frac{D_{0} \tau}{1 \quad i \omega \tau}\left(1+\frac{C_{1}}{1} i \omega \tau\right)+\sum_{j 1}^{3} \frac{i F_{j} \omega}{\left(\omega^{2} \quad \omega_{0 j}^{2}\right)+i \omega \gamma_{j}}$

where $D_{0}$ is the Drude weight, $\tau$ is momentum relaxation time, $F_{j}$ is the oscillator strength of the $j$ 'th oscillator with angular frequency $\omega_{0 j}$ and lifetime broadening parameter $\gamma_{j}$. The first term is the Drude Smith part for the contribution of the carriers to the conductivity where $C_{1}\left(1 \leqslant C_{1} \leqslant 0\right)$ is related to the degree of localization of the carriers; $C_{1} \quad 1$ corresponds to complete localization and $C_{1} \quad 0$ is the Drude limit giving free carrier response. The fitted curves shown by the red and blue solid lines in Fig. 3b, following Eq. (2) repro duce the conductivity spectra very well. The best fit is obtained by using the parameter $C_{1} \quad 0.947 \pm 0.004$, indicat ing a significant degree of carrier localization. As mentioned above, the $s p^{2}$ hybridized carbon regions are separated by the $s p^{3}$ matrix [37] resulting in a potential barrier between the adjacent $s p^{2}$ regions. The carriers can either cross the bar nier to reach the adjacent $s p^{2}$ domains, or they can backscat ter resulting in carrier localization. The $C_{1}$ parameter takes into account the relative probabilities of these two extremes [38]. In case of $s p^{2}$ domains fully isolated by large $s p^{3}$ regions, the probability of reflection is high giving $\left|C_{1}\right| \sim 1$. If there are other $s p^{2}$ clusters in the immediate vicinity, the carriers with sufficient energy may cross the $s p^{2}$ domain boundaries by either hopping or tunneling processes, resulting in $C_{1}$ between 0 and 1 . We further note that the fitted value of the momentum relaxation time $\tau$ is as small as $6.7 \pm 0.5 \mathrm{fs}$. 
We now estimate the Fermi energy $E_{F}$ by extrapolating fit ted real part of conductivity to $\omega \quad 0\left(\begin{array}{ll}\sigma_{D C} & 2.9 G_{0}\end{array}\right)$. Since the RGO membrane consists of randomly stacked graphene lay ers, we can assume that the carriers are distributed equally in each layer. As a consequence, the DC conductivity can be expressed [23] as $\sigma_{D C} \quad \tau \frac{v_{F} e^{2}}{\pi h} \sqrt{ } n \pi N$, where $n$ is carrier density in each layer and $N$ is the number of graphene layers. The approximate number of graphene layers in the $\mathrm{RGO}_{\mathrm{HZ}}$ mem brane is $N \sim 370 / .356 \approx 1040$. Further, the carrier density $n$ is related to Fermi energy $E_{F}$ by $E_{F} \quad \hbar v_{F} \sqrt{ } n \pi$. By taking $\tau \quad 6.7$ fs and $\sigma_{D C} \quad 2.9 G_{0}$, we get Fermi energy to be $\sim 8 \mathrm{meV}$.

Now we focus to understand the three resonances located at $v_{1} \sim 1.8 \mathrm{THz}(7.4 \mathrm{meV}), v_{2} \sim 2.2 \mathrm{THz}(9.0 \mathrm{meV})$ and $v_{3} \sim 2.7 \mathrm{THz}(11.0 \mathrm{meV})$. There are recent theoretical studies on low frequency $\left(<150 \mathrm{~cm}^{1}\right)$ phonon modes in layered crys tals (such as a few layer Bernal stacked graphene and $h \mathrm{BN}$ ) associated with the movements of planes perpendicular to the hexagonal axis (shear modes) and parallel to the hexag onal axis (compression modes) [39]. Some of these modes have been observed recently by femto second coherent pho non spectroscopy [40] and by X ray scattering [41]. The three resonances observed by us in the RGO membrane, though very close to the calculated values of the compression modes for a few layer graphene, may not be associated with these phonons because the graphene layers in RGO do not have perfect Bernal stacking, as assumed in the theoretical calculations [39]. Recently, terahertz conductivity of twisted bilayer graphene has shown a resonance in real part of $\sigma(\omega)$ at $\sim 2.7 \mathrm{THz}[28]$. This has been assigned to an enhanced density of electronic states from the van Hove singularities (VHS) arising from the two twisted graphene layers. We make a similar assignment: The RGO membrane is expected to have graphene layers stacked in turbostratic manner [42]. The rotation between the layers gives rise to Moiré Pattern and hence a superlattice on the graphite surface [29]. For simplicity we consider a graphene bilayer. In Bernal stacking A1 (B1) atom of layer 1 sits on B2 (A2) atom of layer 2 and B1 (A2) atom is at the middle of layer 2 (1). The condition for commensurate rotation by an angle $\theta$ about B2 (A1) to pro duce Moiré pattern is given by [29] $\cos \left(\theta_{j}\right) \frac{3 j^{2}+3 j+1 / 2}{3 j^{2}+3 j+1}$ where $j$ being an integer. $j 0, \theta 60^{\circ}$ corresponds to AA stacked and $j \rightarrow \infty, \theta \quad 0^{\circ}$ corresponds to $A B$ stacked bilayer. The rel ative rotation of the layers causes a shift between Dirac points in momentum space by $\Delta K \quad 2 K \sin (\theta / 2)$ with K $4 \pi / 3 a_{0}$ where $a_{0} \quad 2.46 \AA$. The two Dirac cones intersect near middle of the superlattice Brillouin zone and hybridize resulting in saddle point in energy dispersion and VHSs in the density of states. The hybridized states near zero energy result from the coupling of the zero energy states of one layer with states of energy $\pm \hbar v_{F} \Delta K$ in other layer with inter layer hopping amplitude [43], $t_{\perp}^{\theta} \approx 0.4 t_{\perp}$. It is important to note that the cases where $t_{\perp}^{\theta} \quad 0$ but $\theta \neq 0$, the VHS do not appear even though Moiré patterns can form [29]. For small angle of rotation the energy difference between the VHSs is given by $\Delta E_{\mathrm{VHS}} \frac{8 \pi h v_{\mathrm{F}}}{3 a_{0}}|\sin (\theta / 2)| 2 t_{\perp}^{\theta}$. Thus $\Delta E_{\mathrm{VHS}}$ can be easily tuned by changing the twisting angle, the interlayer coupling and strain [44]. The rotation induced generation of VHS is not limited to graphene bilayer but also to multi layer graphene, provided $t_{\perp}^{\theta} \neq 0$. The value of $t_{\perp}^{\theta}$ is close to $0.1 \mathrm{eV}$ [45]. Taking $\Delta E_{\mathrm{VHS}}$ as the three observed resonances, a consistence choice of the parameters are: $j \quad 28, \theta \quad 1.16$ and $t_{\perp}^{\theta} \quad 0.110,0.109$ and $0.108 \mathrm{eV}$, corresponding to the observed resonances at 1.8, 2.2 and $2.7 \mathrm{THz}$, respectively. A small spread in the value of $t_{\perp}^{\theta}$ can arise from the small var iation in the interlayer spacing.

To see the effect of film thickness and reduction condition, we have measured the terahertz conductivity of $\mathrm{RGO}_{\mathrm{HI}}$. It can be seen from Fig. $3 c$ that the two electronic resonances occur at 1.5 and $2.3 \mathrm{THz}$ which are again due to interband transition across band gap arising from rotation induced generation of VHS in density of states. The zero frequency conductivity of $\mathrm{RGO}_{\mathrm{HI}}$ in unit of $\mathrm{S} / \mathrm{m}$ is $500 \mathrm{~S} / \mathrm{m}$ which is similar to $600 \mathrm{~S} / \mathrm{m}$ in $\mathrm{RGO}_{\mathrm{Hz}}$. The value of the momentum relaxation time $\tau$ is $98 \pm 1$ fs and the parameter $C_{1} \quad 0.77 \pm 0.01$ which corroborate the fact that the oxygen functional groups in $\mathrm{RGO}_{\mathrm{HI}}$ are less that in $\mathrm{RGO}_{\mathrm{Hz}}[32]$.

\subsection{Optical pump - terahertz probe studies}

We now investigate the changes in terahertz conductivity by optical pump using time resolved optical pump terahertz probe spectroscopy. To measure the photoinduced $\mathrm{THz}$ elec tric field transmission, we follow the procedure shown in Fig. 2. The sample is photoexcited with $785 \mathrm{~nm} \mathrm{(} \mathrm{70} \mathrm{fs}$ pulses) pump. The gate line is kept fixed at the peak of the $\mathrm{THz}$ electric field $\left(t_{d} \quad 0\right)$. The pump induced change of the peak of $\mathrm{THz}$ waveform is measured by changing the delay line $t_{p}$ of optical pump in a way such that the optical pump reaches the sample earlier than the $\mathrm{THz}$ probe. The photoin duced change in terahertz transmission: $\Delta \mathrm{T} \quad \mathrm{T}$ (pump on) $\mathrm{T}$ (pump off), is related to photoinduced conductivity $\Delta \sigma$ by the relation $\frac{\Delta T}{T} \approx \frac{Z_{0}}{2} \Delta \sigma$. Now we will present the results of $\mathrm{RGO}_{\mathrm{HZ}}$. Fig. $4 \mathrm{a}$ shows the photoinduced change of conductiv ity measured at the peak of the terahertz electric field $\left(\begin{array}{ll}t_{d} & 0\end{array}\right)$ as a function of the delay time between optical pump and terahertz probe pulses using the pump fluence of $238 \mu \mathrm{J} / \mathrm{cm}^{2}$. Here the photoconductivity $\Delta \sigma$ is positive, simi lar to earlier reports on single layer graphene [22,46]. We must note that negative $\Delta \sigma$ has also been observed in graph ene $[16,17,47,48]$ and tuning of $\Delta \sigma$ has been addressed in recent studies $1[16,17,49]$. The positive $\Delta \sigma$ is due to domi nance of intraband scattering of photoinduced carriers over the other cooling channel involving secondary hot carrier generation 1[47]. $\Delta \sigma$ can be related to corresponding electron temperature $\mathrm{T}_{e}$ by using

$$
\begin{aligned}
& \Delta \sigma \quad \sigma_{\text {intra }}\left(\mathrm{T}_{e}\right) \quad \sigma_{\text {intra }}\left(\mathrm{T}_{0}\right) \\
& \sigma_{\text {intra }}(\mathrm{T}) \quad \frac{2 \mathrm{G}_{0} \tau k_{\mathrm{B}} \mathrm{T}}{\hbar\left(\omega^{2} \tau^{2}+1\right)} \ln \left[2 \cosh \left(\frac{E_{\mathrm{F}}}{2 k_{\mathrm{B}} \mathrm{T}}\right)\right]
\end{aligned}
$$

Here $T_{0}(300 \mathrm{~K})$ is the lattice temperature. The value of $\tau$ is taken $30 \mathrm{fs}$ obtained from fitting the frequency dependence of the dynamic conductivity (to be shown later). Fig. $4 \mathrm{~b}$ shows the fluence dependence of $T_{e}^{\max }$ corresponding to $\Delta \sigma^{\max }$. The solid line is a fit to the equation $\mathrm{T}_{e}^{\max }\left(\mathrm{T}_{0}^{3}+B \varphi\right)^{1 / 3}[50,51]$ whose origin is in the linear dependence of electronic density of states on energy as in graphene. The best fit is obtained for B $6.7 \times 10^{7} \mathrm{~J}^{1} \mathrm{~m}^{2} \mathrm{~K}^{3}$.

We now focus on the cooling dynamics of the hot carriers. The decay of $\Delta \sigma$ consists of three components; a fast relaxa 

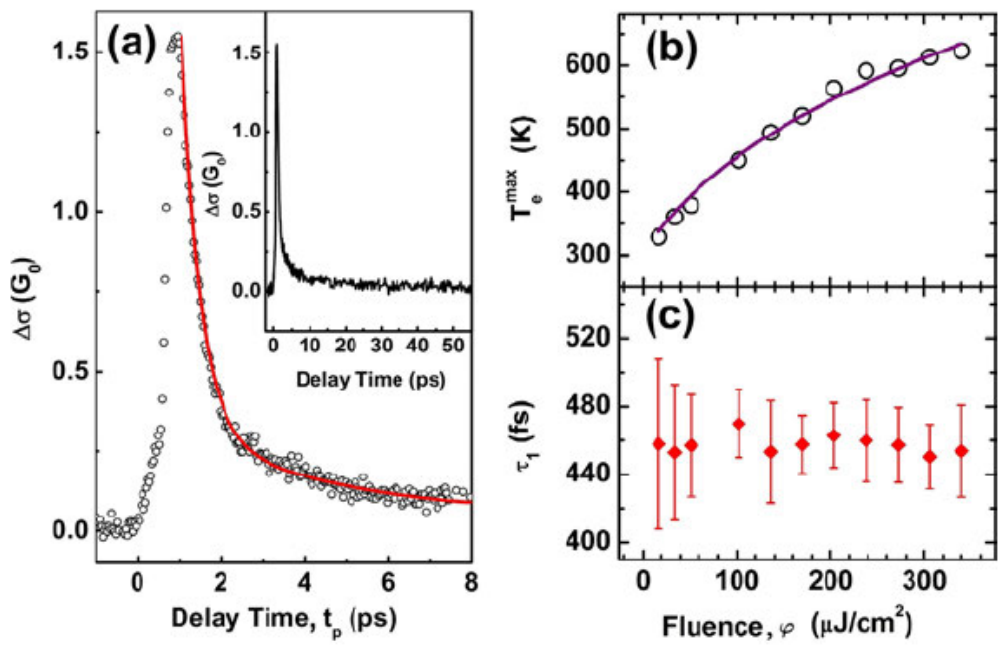

Fig. 4 - (a) Photoinduced change of Terahertz conductivity $\Delta \sigma$ of $\mathrm{RGO}_{\mathrm{Hz}}$. The inset shows the decay dynamics up to 55 ps. The solid red line in the main panel is a fit to super-collision model including the fast relaxation component $\tau_{1}$. (b) $T_{e}^{\max }$ plotted as a function of pump fluence. Solid line is fit to function $\left(T_{0}^{3}+B \varphi\right)^{1 / 3}$. (c) The fast decay component $\tau_{1}$ is displayed as function of $\varphi$. The fitted $\tau_{1}$ shows $\varphi$ independent behavior. (A color version of this figure can be viewed online.)

tion with time constant $\tau_{1}$ (a few hundred fs), a slow compo nent $\tau_{2}$ (a few ps) and a small third component with long decay time $\tau_{3}$. The origin of $\tau_{1}$ can be Auger recombination [30] or optical phonon emission [31,50]. For the former pro cess, $\tau_{1}$ should decrease with $\varphi$ as observed in graphene oxide. This is however not the case in the present study in Fig. 4c. Thus, $\tau_{1}$ can be associated with the optical phonon emission instead of Auger recombination. This difference from Ref. [30] may arise due to different method of reducing GO (Ref. [30] has used thermal annealing as compared to chemical reduction by us).

The electron optical phonon scattering continues till the hot carriers energy is reduced to less than the optical phonon energy ( $200 \mathrm{meV})$. Below $200 \mathrm{meV}$, emission of acoustic pho nons is the only possible way of energy relaxation permitted by momentum conservation. At each scattering event, very small energy $\left(k_{B} T_{B G} 2 v_{S} E_{F} / v_{F}, v_{s}\right.$ is sound velocity) is dissipated and thus the cooling process can last as long as $300 \mathrm{ps}$ [46]. Here, $\mathrm{T}_{B G}$ is Bloch Gruneisen temperature defining a boundary between the low and the high temperature response. A recent proposal for the relaxation process with time constant $\tau_{2}$ is the emission of high energy $\left(\sim k_{B} T_{e}\right)$ and high momentum $\left(\sim k_{\mathrm{B}} \mathrm{T}_{e} / h v_{s}\right)$ acoustic phonons mediated by disorder (for wave vector conservation) and is termed as super collision cooling (SC) with relaxation time of a few ps [52]. For the SC mechanism the rate equation governing the cooling of the carriers is given by [52 54]

$\frac{\partial \mathrm{T}_{e}(\mathrm{t})}{\partial \mathrm{t}} \quad \frac{\mathrm{A}}{\alpha} \frac{\mathrm{T}_{e}^{3}(\mathrm{t}) \quad \mathrm{T}_{0}^{3}}{\mathrm{~T}_{e}(\mathrm{t})}$

Here $A / \alpha$ is the SC rate coefficient which is related to defor mation potential, $D_{a c}$ by $[53,54]$

$\frac{A}{\alpha} \quad \frac{6 \zeta(3)}{\pi^{2}} \frac{g}{k_{F} \ell} \frac{k_{B}}{h} \cong \frac{2}{3} \frac{g}{k_{F} \ell} \frac{k_{B}}{h}$

where $g$, electron phonon coupling strength is $2 D_{a c}^{2} E_{F} / \rho v_{s}^{2} \pi$ $\left(h v_{\mathrm{F}}\right)^{2}, \zeta$ is Riemann zeta function and $\rho \sim 7.6 \times 10^{7} \mathrm{~kg} / \mathrm{m}^{2}$ is density of graphene. The fitted curve shown by solid line in Fig. 4a consists of the initial decay, taken in the form $A_{1} \exp \left(t / \tau_{1}\right)$ to represent electron phonon scattering process along with decay of $T_{e}$ due to SC mechanism given by Eq. (4). The best fit is obtained using $A_{1} \quad 0.78, \tau_{1} \quad 450 \mathrm{fs}, \tau \quad 30 \mathrm{fs}$ and $A / \alpha \quad 2.6 \times 10^{8} \mathrm{~K}^{1} \mathrm{~s}^{1}$. The value of $\mathrm{A} / \alpha$ is very close to the reported rate coefficient for the suspended graphene [53]. Using Eq. (5) the deformation potential $D_{a c}$ is $\sim 15 \mathrm{eV}$, which is close to the reported experimental values [55,56].

A very slow relaxation time of $\tau_{3} \sim 100 \mathrm{ps}$ is observed in the relaxation dynamics $\left[A_{3} \exp \left(t / \tau_{3}\right)\right.$ with $\left.A_{3} \quad 0.02\right]$. This term can be related to the trapping of the electrons in deep trap states of $\mathrm{RGO}_{\mathrm{Hz}}$ and was also observed by transient absorption measurements [31]. The trap density $N_{t}$ can be estimated from $\tau_{3}$ by using $N_{t}{ }^{1} \quad \tau_{3} B v_{F}$ where $B$ is the absorption cross section [57] taken as $\sim 10^{14} \mathrm{~cm}^{2}$. The estimated trap density $N_{t}$ is thus $9 \times 10^{15} / \mathrm{cm}^{3}$, close to the trap densities observed by current voltage measurements [58,59]. The OPTP measurements on $\mathrm{RGO}_{\mathrm{HI}}$ show similar relaxation dynamics and fluence dependence.

We now measure the frequency dependence of $\Delta \sigma(\omega)$ at different times of carrier relaxation. At time $t_{p}$ after photoex citation, the THz pulse was sent through the membrane. The mapping of the photoinduced change in transmission $(\Delta T)$ of the THz waveform in time domains was done by varying the arrival time of the gate pulse and the $\mathrm{THz}$ pulse using the time delay $t_{d}$. The mapped $\Delta T$ is shown in Fig. 5 . Since the gate line was used, each point of the terahertz waveform experi ences a different delay after photoexcitation and thus probe the $\mathrm{RGO}_{\mathrm{Hz}}$ system in different states over a time scale of the duration of the $\mathrm{THz}$ waveform. Thus, the information recorded is an average of the non equilibrium dynamics of the hot carriers over a few ps. The complex $\Delta \sigma(\omega)$, extracted from $\Delta \mathrm{T}\left(\mathrm{t}_{d}\right)$ at different $t_{p}$ after photoexcitation are shown in Fig. 6. The real part of $\Delta \sigma$ is positive, increasing with frequency while the imaginary part is completely negative and decreases with frequency. The $\Delta \sigma(\omega)$ is well described 


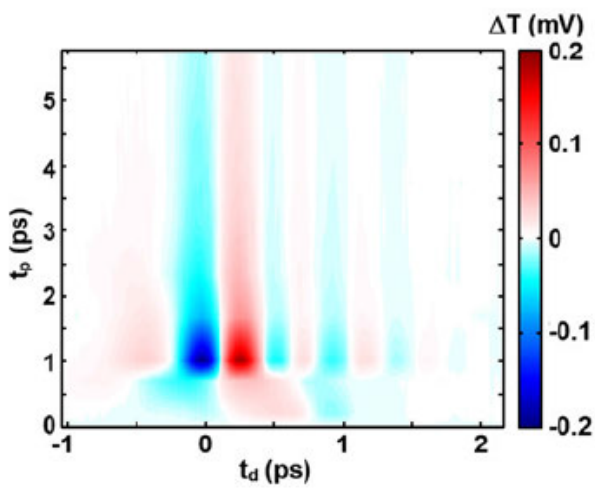

Fig. 5 - Mapping of the Photo-induced change of THz transmission $(\Delta \mathrm{T})$. (A color version of this figure can be viewed online.)

by Drude Smith model (given by the first term in Eq. (2)) after $t_{p} 3$ ps. Below 3 ps the model does not fit well as expected due to very high rate of carrier relaxation just after achieving the quasi equilibrium condition. The fitted parameter, momentum relaxation time $\tau$ is $30 \pm 2 \mathrm{fs}$ (and is independent of $\left.t_{p}\right)$ and $\left|C_{1}\right|$ increases with $t_{p}$ as shown in Fig. 7a. The increase of $\tau$ from 6.7 to $30 \mathrm{fs}$ after photoexcitation can arise since the hot carriers have enough energy to cross the nearby barriers resulting in higher average momentum relaxation time. Further, the increase of $\left|C_{1}\right|$ with $t_{p}$ confirms the same. The extracted Drude weight $\left(D_{0}\right)$ is plotted in Fig. $7 \mathrm{~b}$ as a func tion of $t_{p}$. The red solid line is a fit to the function $D_{0} \quad D_{0}^{\circ}+D_{t} \exp \left(\frac{t_{p} t_{0}}{\tau_{D}}\right)$ with $t_{0} 1 p s$ (corresponds to the maximum in OPTP signal shown in Fig. 4a) and the fitted parameters are $D_{0}^{\circ} 2.0 \times 10^{9} \Omega{ }^{1} \mathrm{~s}^{1}, D_{\mathrm{t}} \quad 1.1 \times 10^{10} \Omega{ }^{1} \mathrm{~s}^{1}$ and

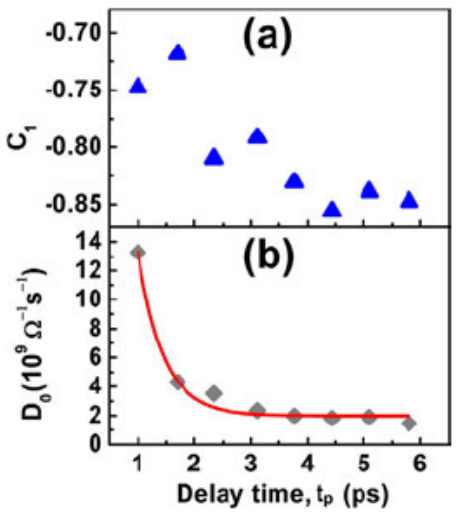

Fig. 7 - Fitted parameters (a) $C_{1}$ and (b) $D_{0}$ are plotted as a function of $t_{p}$. The red solid line is the fit to the exponential function with time constant $\sim \mathbf{4 5 0}$ fs as described in the text. (A color version of this figure can be viewed online.)

$\tau_{\mathrm{D}} \quad 450 \mathrm{fs}$. The time constant $\tau_{\mathrm{D}}$ is same as $\tau_{1}$ due to elec tron optical phonon scattering processes.

\section{Conclusions}

Terahertz time domain measurements of two kinds of RGO membranes are carried out to measure the conductivity from 0.5 to $3.5 \mathrm{THz}$. The terahertz conductivity spectra reveal devi ation from the Drude form and are explained in terms of Drude Smith model which takes into account the back scat tering of the carriers. In addition, three resonances are observed in the conductivity spectra of $\mathrm{RGO}_{\mathrm{Hz}}$. The origin of these resonances is best attributed to the enhanced density of states near the Dirac cone due to rotation of adjacent
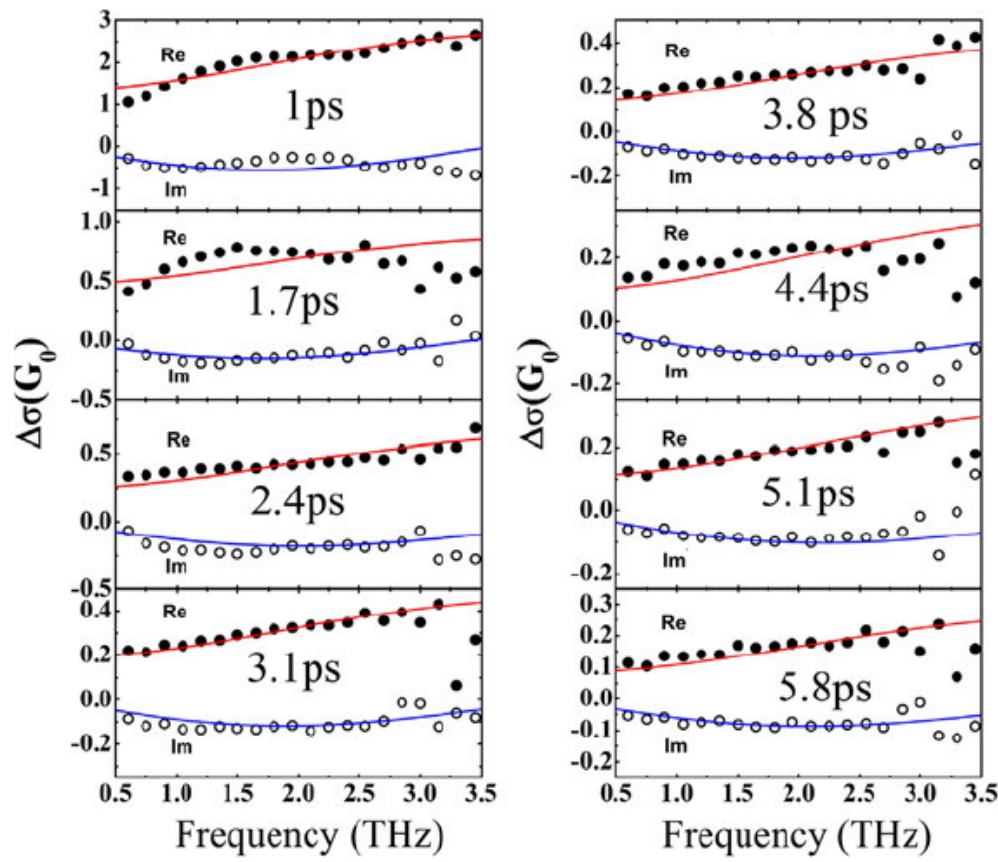

Fig. 6-Spectral dependence of photo-induced dynamic conductivity $\Delta \sigma(\omega)$ at different delay time $t_{p}$. The real part is shown by closed circles and imaginary part by open circles. The solid lines are fit to the Drude-Smith model. (A color version of this figure can be viewed online.) 
graphene layers. A control on the relative orientation of the layers in graphene is important to tune the electronic struc ture of RGO. Photoexcited dynamics was studied by optical pump terahertz probe spectroscopy where a positive value of dynamic photoconductivity is observed that can be explained by intraband scattering processes. The cooling dynamics is explained in term of fast cooling involving optical phonons along with the super collision cooling involving acoustic phonons and disorder. The fitted parameters of super collision cooling model are used to estimate the defor mation potential $(\sim 15 \mathrm{eV})$. We hope that the present study will help in evaluating the application potential of RGO for ultrafast opto electronic applications. It will be interesting to study reduced graphene oxide with systematic quantitative variation of degree of oxygen functionalities.

\section{Acknowledgement}

AKS thanks the Department of Science and Technology for financial assistance under the Nanomission Project and Gyan Prakash for his initial involvement in the project.

\section{R E F E R E N C E S}

[1] Bonaccorso F, Sun Z, Hasan T, Ferrari AC. Graphene photonics and optoelectronics. Nat Photonics 2010;4:611 22.

[2] Novoselov KS, Geim AK, Morozov SV, Jiang D, Katsnelson MI, Grigorieva IV, et al. Two dimensional gas of massless dirac fermions in graphene. Nature 2005;438(7065):197 200.

[3] Morozov SV, Novoselov KS, Katsnelson MI, Schedin F, Elias DC, Jaszczak JA, et al. Giant intrinsic carrier mobilities in graphene and its bilayer. Phys Rev Lett 2008;100:016602.

[4] Avouris P. Graphene: electronic and photonic properties and devices. Nano Lett 2010;10(11):4285 94.

[5] Geim AK, Novoselov KS. The rise of graphene. Nat Mater 2007;6:183 91.

[6] Weis P, Garcia Pomar JL, Hh M, Reinhard B, Brodyanski A, Rahm M. Spectrally wide band terahertz wave modulator based on optically tuned graphene. ACS Nano 2012;6(10):9118 24.

[7] Bao Q Loh KP. Graphene photonics, plasmonics, and broadband optoelectronic devices. ACS Nano 2012;6(5):3677 94.

[8] Liu M, Yin X, Ulin Avila E, Geng B, Zentgraf T, Ju L, et al. Graphene based broadband optical modulator. Nature 2011;474:64 7.

[9] Miao X, Tongay S, Petterson MK, Berke K, Rinzler AG, Appleton BR, et al. High efficiency graphene solar cells by chemical doping. Nano Lett 2012;12(6):2745 50.

[10] Shi E, Li H, Yang L, Zhang L, Li Z, Li P, et al. Colloidal antireflection coating improves graphene silicon solar cells. Nano Lett 2013;13(4):1776 81.

[11] Sun Z, Hasan T, Torrisi F, Popa D, Privitera G, Wang F, et al. Graphene mode locked ultrafast laser. ACS Nano 2010;4(2):803 10.

[12] Xia F, Mueller T, Lin Ym, Valdes Garcia A, Avouris P. Ultrafast graphene photodetector. Nat Nanotechnol 2009;4:839 43.

[13] Cravotto G, Cintas P. Sonication assisted fabrication and post synthetic modifications of graphene like materials. Chem Eur J 2010;16:5246 59.
[14] Li D, Müller MB, Gilje S, Kaner RB, Wallace GG. Processable aqueous dispersions of graphene nanosheets. Nat Nanotechnol 2008;3:101 5.

[15] Huang N, Lim H, Chia C, Yarmo M, Muhamad M. Simple room temperature preparation of high yield large area graphene oxide. Int J Nanomed 2011;6:3443 8.

[16] Jnawali G, Rao Y, Yan H, Heinz TF. Observation of a transient decrease in terahertz conductivity of single layer graphene induced by ultrafast optical excitation. Nano Lett 2013;13(2):524 30.

[17] Frenzel AJ, Lui CH, Fang W, Nair NL, Herring PK, Jarillo Herrero P, et al. Observation of suppressed terahertz absorption in photoexcited graphene. Appl Phys Lett 2013;102(11):113111.

[18] Baek IH, Ahn KJ, Kang BJ, Bae S, Hong BH, Yeom DI, et al. Terahertz transmission and sheet conductivity of randomly stacked multi layer graphene. Appl Phys Lett 2013;102(19):191109.

[19] Li J, Ren Z, Zhou Y, Wu X, Xu X, Qi M, et al. Scalable synthesis of pyrrolic $n$ doped graphene by atmospheric pressure chemical vapor deposition and its terahertz response. Carbon 2013;62(0):330 6 .

[20] Hong JT, Lee KM, Son BH, Park SJ, Park DJ, Park JY, et al. Terahertz conductivity of reduced graphene oxide films. Opt Express 2013;21(6):7633 40.

[21] Dawlaty JM, Shivaraman S, StraitJ, George P, Chandrashekhar M, Rana F, et al. Measurement of the optical absorption spectra of epitaxial graphene from terahertz to visible. Appl Phys Lett 2008;93(13):131905.

[22] Choi H, Borondics F, Siegel DA, Zhou SY, Martin MC, Lanzara A, et al. Broadband electromagnetic response and ultrafast dynamics of few layer epitaxial graphene. Appl Phys Lett 2009;94(17):172102.

[23] Yan H, Xia F, Zhu W, Freitag M, Dimitrakopoulos C, Bol AA, et al. Infrared spectroscopy of wafer scale graphene. ACS Nano 2011;5(12):9854 60.

[24] Obraztsov PA, Rybin MG, Tyurnina AV, Garnov SV, Obraztsova ED, Obraztsov AN, et al. Broadband light induced absorbance change in multilayer graphene. Nano Lett 2011;11(4):1540 5.

[25] Maeng I, Lim S, Chae SJ, Lee YH, Choi H, Son JH. Gate controlled nonlinear conductivity of dirac fermion in graphene field effect transistors measured by terahertz time domain spectroscopy. Nano Lett 2012;12(2):551 5.

[26] Ren L, Zhang Q, Yao J, Sun Z, Kaneko R, Yan Z, et al. Terahertz and infrared spectroscopy of gated large area graphene. Nano Lett 2012;12(7):3711 5.

[27] Badhwar S, Sibik J, Kidambi PR, Beere HE, Axel Zeitler J, Hofmann S, et al. Intrinsic terahertz plasmon signatures in chemical vapour deposited graphene. Appl Phys Lett 2013;103(12):121110.

[28] Zou X, Shang J, Leaw J, Luo Z, Luo L, La o vorakiat C, et al. Terahertz conductivity of twisted bilayer graphene. Phys Rev Lett 2013;110:067401.

[29] Li G, Luican A, Lopes dos Santos JMB, Castro Neto AH, Reina A, Kong J, et al. Observation of van hove singularities in twisted graphene layers. Nat Phys 2010;6:109 13.

[30] Kim J, Oh J, In C, Lee YS, Norris TB, Jun SC, et al. Unconventional terahertz carrier relaxation in graphene oxide: observation of enhanced auger recombination due to defect saturation. ACS Nano 2014;8(3):2486 94.

[31] Kaniyankandy S, Achary SN, Rawalekar S, Ghosh HN. Ultrafast relaxation dynamics in graphene oxide: evidence of electron trapping. J Phys Chem C 2011;115(39):19110 6.

[32] Moon IK, Lee J, Ruoff RS, Lee H. Reduced graphene oxide by chemical graphitization. Nat Commun 2010;1:73.

[33] Cancado LG, Jorio A, Ferreira EHM, Stavale F, Achete CA, Capaz RB, et al. Quantifying defects in graphene via raman 
spectroscopy at different excitation energies. Nano Lett 2011;11(8):3190 6.

[34] Cook DJ, Hochstrasser RM. Intense terahertz pulses by four wave rectification in air. Opt Lett 2000;25(16):1210 2.

[35] Grischkowsky D, Keiding S, van Exter M, Fattinger C. Far infrared time domain spectroscopy with terahertz beams of dielectrics and semiconductors. J Opt Soc Am B 1990;7(10):2006 15.

[36] Duvillaret L, Garet F, Coutaz JL. Influence of noise on the characterization of materials by terahertz time domain spectroscopy. J Opt Soc Am B 2000;17(3):452 61.

[37] Loh KP, Bao Q, Eda G, Chhowalla M. Graphene oxide as a chemically tunable platform for optical applications. Nat Chem 2010;2:1015 124.

[38] Titova LV, Cocker TL, Cooke DG, Wang X, Meldrum A, Hegmann FA. Ultrafast percolative transport dynamics in silicon nanocrystal films. Phys Rev B 2011;83:085403.

[39] Michel KH, Verberck B. Theory of rigid plane phonon modes in layered crystals. Phys Rev B 2012;85:094303.

[40] Boschetto D, Malard L, Lui CH, Mak KF, Li Z, Yan H, et al. Real time observation of interlayer vibrations in bilayer and few layer graphene. Nano Lett 2013;13(10):4620 3.

[41] Mohr M, Maultzsch J, Dobardi E, Reich S, Miloevi I, Damnjanovi M, et al. Phonon dispersion of graphite by inelastic X ray scattering. Phys Rev B 2007;76:035439.

[42] Gmez Navarro C, Meyer JC, Sundaram RS, Chuvilin A, Kurasch S, Burghard M, et al. Atomic structure of reduced graphene oxide. Nano Lett 2010;10(4):1144 8.

[43] Lopes dos Santos JMB, Peres NMR, Castro Neto AH. Continuum model of the twisted graphene bilayer. Phys Rev B 2012;86:155449.

[44] Chu ZD, He WY, He L. Coexistence of van hove singularities and superlattice dirac points in a slightly twisted graphene bilayer. Phys Rev B 2013;87:155419.

[45] Xing G, Guo H, Zhang X, Sum TC, Huan CHA. The physics of ultrafast saturable absorption in graphene. Opt Express 2010;18(5):4564 73.

[46] Strait JH, Wang H, Shivaraman S, Shields V, Spencer M, Rana F. Very slow cooling dynamics of photoexcited carriers in graphene observed by optical pump terahertz probe spectroscopy. Nano Lett 2011;11(11):4902 6.

[47] Tielrooij KJ, Song JCW, Jensen SA, Centeno A, Pesquera A, Zurutuza Elorza A, et al. Photoexcitation cascade and multiple hot carrier generation in graphene. Nat Phys 2013;9:248 52.

[48] Docherty CJ, Lin CT, Joyce HJ, Nicholas RJ, Herz LM, Li LJ, et al. Extreme sensitivity of graphene photoconductivity to environmental gases. Nat Commun 2012;3:1228.

[49] Shi SF, Tang TT, Zeng B, Ju L, Zhou Q, Zettl A, et al. Controlling graphene ultrafast hot carrier response from metal like to semiconductor like by electrostatic gating. Nano Lett 2014;14(3):1578 82.

[50] Gierz I, Petersen JC, Mitrano M, Cacho C, Turcu ICE, Springate $E$, et al. Snapshots of non equilibrium dirac carrier distributions in graphene. Nat Mater 2013;12:1119 24.

[51] Winzer T, Mali E. Impact of auger processes on carrier dynamics in graphene. Phys Rev B 2012;85:241404.

[52] Song JCW, Reizer MY, Levitov LS. Disorder assisted electron phonon scattering and cooling pathways in graphene. Phys Rev Lett 2012;109:106602.

[53] Graham MW, Shi SF, Ralph DC, Park J, McEuen PL. Photocurrent measurements of supercollision cooling in graphene. Nat Phys 2013;9:103 8.

[54] Betz AC, Jhang SH, Pallecchi E, Ferreira R, Feve G, Berroir JM, et al. Supercollision cooling in undoped graphene. Nat Phys 2013;9:109 12.

[55] Bolotin KI, Sikes KJ, Hone J, Stormer HL, Kim P. Temperature dependent transport in suspended graphene. Phys Rev Lett 2008; 101:096802.

[56] Dean CR, Young AF, Meric I, Lee C, Wang L, Sorgenfrei S, et al. Boron nitride substrates for high quality graphene electronics. Nat Nanotechnol 2010;5:722 6.

[57] Schneck JR, Walsh AG, Green AA, Hersam MC, Ziegler LD, Swan AK. Electron correlation effects on the femtosecond dephasing dynamics of e22 excitons in $(6,5)$ carbon nanotubes. J Phys Chem A 2011;115(16):3917 23. http:// dx.doi.org/10.1021/jp108345t.

[58] Joung D, Chunder A, Zhai L, Khondaker SI. Space charge limited conduction with exponential trap distribution in reduced graphene oxide sheets. Appl Phys Lett 2010;97(9):093105.

[59] Kajen RS, Chandrasekhar N, Pey KL, Vijila C, Jaiswal M, Saravanan S, et al. Charge transport in lightly reduced graphene oxide: a transport energy perspective. J Appl Phys 2013;113(6):063710. 